\title{
Acute oral toxicity of zein nanoparticles with encapsulated gamma oryzanol in Sprague Dawley rats
}

\author{
Ubonphan Rodsuwan ${ }^{1}$, Krittiya Thisayakorn ${ }^{2}$, Benjawan Thumthanaruk ${ }^{1}$, Savitri Vatanyoopaisarn ${ }^{1}$, Dudsadee Uttapap $^{3}$, \\ Qixin Zhong ${ }^{4}$, and Vilai Rungsardthong ${ }^{1, *}$
}

\begin{abstract}
${ }^{1}$ Department of Agro-Industrial, Food and Environmental Technology, Faculty of Applied Science, Food and Agro-Industrial Research Center, King Mongkut's University of Technology North Bangkok, 1518, Pracharat 1 Road, Bangsue, Bangkok 10800, Thailand ${ }^{2}$ Expert Center of Innovative Herbal Products (InnoHerb), Thailand Institute of Scientific and Technological Research (TISTR), Khlong Luang, Pathum Thani 12120, Thailand

${ }^{3}$ Division of Biochemical Technology, School of Bioresources and Technology, King Mongkut's University of Technology Thonburi, Bangkhuntian Bangkok 10150, Thailand

${ }^{4}$ Department of Food Science, The University of Tennessee, 2510 River Drive, Knoxville, TN 37996, United States
\end{abstract}

\begin{abstract}
Gamma oryzanol (GO) is well known for its antioxidant activity and health-promoting benefits. The gamma oryzanol-loaded zein nanoparticles (GOZNs) were successfully prepared in our previous study. In the present work, the acute oral toxicity of GOZNs was evaluated based on OECD guideline 420. GOZNs were fabricated by the liquid-liquid dispersion and lyophilized. The samples displayed a mean diameter of $311.20 \pm 3.01 \mathrm{~nm}$ and high loading capacity of $311.22 \pm 7.97$ to $322.69 \pm 5.67 \mathrm{mg}-\mathrm{GO} / \mathrm{g}$-powder after 42-day storage at $-18^{\circ} \mathrm{C}$. Healthy female Sprague Dawley rats $(8$ weeks of age) were used for the experiments. Five female rats were administered a single dose of $2,000 \mathrm{mg} \mathrm{GO} / \mathrm{kg}$ body weight via the oral route. Observations of toxicity signs were recorded for the first 24 hours, and the changes in the general physical conditions were monitored for 14 days before the gross necropsy on day 15 . The results show that Sprague Dawley rats exhibited normal growth, and neither mortality nor acute toxicity signs were observed throughout the study period. The findings revealed that GOZNs did not have acute toxicity and were safe when administered orally in Sprague Dawley rats for short periods with LD50 > 2,000 mg/kg.
\end{abstract}

Keyword. Gamma oryzanol, Zein nanocapsules, Encapsulation, Acute oral toxicity, Sprague Dawley rats

\section{Introduction}

Rice is the most widely consumed staple food in Asia, especially in Thailand, where the rice production in 2020 was 18.6 million tonnes [1]. During industrial rice milling process, rice bran was regarded as a by-product $(8-11 \% \mathrm{w} / \mathrm{w})$ and used as low-grade animal feed. Gamma oryzanol (GO) is a mixture of ferulic acid esters with phytosterol and can be extracted from rice bran [2]. The potent antioxidant capacity, inhibition effects on lipid peroxidation, and therapeutically health-promoting functions have been reported for GO. Gamma oryzanol was proved as safe for consumption with no significant side effects [3,4]. However, the applications of GO as a functional ingredient or in food systems have been limited because of its sensitivity to degradation by heat and oxidation, lower bioavailability, and poor watersolubility [5]. Encapsulation of GO within a biopolymeric material could overcome these limitations.

The utilization of nanoscale materials has been increasing exponentially throughout the world in various fields such as pharmaceuticals, cosmetics, and food. Nanoencapsulation is defined in the pharmaceutical area as forming nanometric carriers with a diameter from a few $\mathrm{nm}$ to just below $1000 \mathrm{~nm}$ [6]. Encapsulation is beneficial for targeted delivery, controlled release, and protection of the bioactive components against destructive environments during processing or delivery [7].

Nowadays, many researchers are interested in amphiphilic molecules-based nano-sized drug delivery systems due to their unique core-shell structures and their ability to transport through cell membranes [8]. Zein has been approved for oral use by the FDA and is a safe, non-toxic, biodegradable, and generally recognized as safe (GRAS) biopolymer [6]. More than 50\% amino acid residues of zein consist of proline, glutamine, and asparagine [9]. The tasteless, odourless, distinctive thermal resistance and excellent oxygen barrier characteristics of zein may be the advantages as carrier materials for encapsulating food antioxidants. Moreover, zein is a core biomaterial for delivery systems of hydrophobic nutrients. Zein can self-assemble into different structures, including spheres, sponges, and films [10]. It has been used to encapsule compounds significant to the food, pharmaceutical and textile industries such as tocopherol [11], vitamin D3 [12], flax oil [13], essential oil [14], puerarin [15], sacha inchi oil [16], and fabric softener [17].

\footnotetext{
* Corresponding author: vilai.r@sci.kmutnb.sc.th
} 
GO loaded-zein nanoparticles (GOZNs) were prepared using a liquid-liquid dispersion technology in our previous study [18]. GOZNs can be prepared as a colloidal suspension or dried into powders using several drying technologies such as freeze drying and spray drying, and also provide sustained release in gastrointestinal conditions. However, nanoparticles might cause significant concerns for adverse effects on deliberation to the human body or environment. The toxicity of nanoparticles is closely related to their dose, size, surface area, concentration, chemical properties, and crystalline structure [4]. Nanoparticles could activate biological responses in terms of cellular toxicity and genotoxicity higher than microparticles [19].

The stability, internalization efficiency, and low toxicity are essential requirements for their successful applications. The evaluation of in vivo toxicity of GOZNs is considered essential for their application in food products. Humans have exceedingly complicated metabolic systems. The interactions between each compound in the consumed products and the biological systems could lead to adverse effects, requiring the assessments of the immune response, metabolism patterns, biodistribution, and obliteration of nanoparticles [20]. Pan and Zhong [21] suggested that applications of nanoparticles require in vitro and in vivo studies to guide the development of safe food products that utilize the unique functionalities of nanoparticles. Toxicological studies in animals were raised to identify the possibility of adverse effects from the accumulation of nanoparticles in the body on the immune system, oxidative stress-related disorders, and other diseases $[22,23]$. Subacute toxicity studies of GO have already been performed by Moon and co-workers [24]. Sprague Dawley rats received oral gavage administration of GO at doses of 0,1000 , and $2000 \mathrm{mg} / \mathrm{kg}$ body weight/day survived throughout the study period with no toxicological and histopathological changes. Andersen [25] reported that GO showed no chronic oral toxicity, reproductive toxicity, genotoxicity, and carcinogenicity in vivo. However, to the best of our knowledge, the in vivo toxicology including the acute toxicity of GOZNs has not been reported.

The present study aimed to evaluate the acute oral toxicity of GOZNs in Sprague Dawley rats according to OECD No. 420 [26]. According to literature surveys of conventional LD50 tests in both male and female rats [27], female rats are slightly more sensitive than males. Thus, the female rats-based method in the OECD No. 420 was adopted in this study [26]. The GOZNs were prepared and determined for properties such as particle size, polydispersity index (PDI), and GO content before being fed to the rats. Toxicity signs of each rat were recorded for the first day, and changes in general physical conditions were investigated for 14 days before examining internal organs by gross necropsy on day 15 .

\section{Materials and methods}

\subsection{Materials}

Zein was purchased from Sigma Aldrich Corp., (St. Louis, MO, USA). GO was a product from Tsuno Rice Fine Chemicals Co., Ltd. (Wakayama, Japan). Tween 80 and lecithin were supplies from Union Chemical 1986 Co., Ltd. (Thailand). All other chemicals used were of analytical grade.

\subsection{Preparation of GOZNs and measurement of their properties}

The GOZNs were prepared as described by Rodsuwan and co-worker [18]. Briefly, zein $(0.4 \mathrm{~g})$ was dissolved in $20 \mathrm{ml}$ of $85 \%(\mathrm{w} / \mathrm{v})$ aqueous ethanol with a magnetic stirrer. GO (0.2 g; 50\% loading of zein) was dissolved at $1 \% \mathrm{w} / \mathrm{v}$ in isopropanol that was dispersed dropwise to the zein solution using a magnetic stirrer. The dispersion was added to a citrate solution containing Tween 80 and lecithin with $\mathrm{pH} 8$ to 9 and mixed using a homogenizer (Ultra-Turrax T25, IKA, Germany). The final concentration of GOZNs in dispersion was set at $0.3 \%$ zein and 50\% GO loaded. The resulting mixture was concentrated to $100 \mathrm{ml}$ using a rotary evaporator. The resulting GOZNs dispersions were stored at $4{ }^{\circ} \mathrm{C}$ for further uses. The particle size and PDI of freshly prepared colloidal dispersions were determined by dynamic light scattering using a DelsaTM nanoparticle analyzer (Beckman Coulter, Fullerton, CA). The $\zeta$ potential measurements were performed using a Laser Doppler Velocimeter (Zetasizer Nano ZS90, Malvern, UK). The dispersion was also freeze-dried (Alpha 1-4 LSCplus, Christ, Germany).

\subsection{Morphological observation}

The morphology of GOZNs was investigated using scanning electron microscopy SEM (JSM-6610LV, JEOL, Japan) and FESEM (JSM-7610F, JEOL, Japan). Prior to the observation, the lyophilized GOZNs powder was placed on the stubs and subsequently coated with a thin layer of gold using a sputter coater to avoid charging under the electron beam.

\subsection{Determination of GO content in GOZNs}

High performance liquid chromatography (HPLC) was used to quantify the amount of GO content in GOZNs following a protocol previously described by Rodsuwan and co-workers [18]. Briefly, GOZNs were dissolved in $4 \mathrm{ml}$ of a mixture of methanol and dichloromethane $(1: 1$ $\mathrm{v} / \mathrm{v})$ and filtered through a $0.45 \mu \mathrm{m}$ filter. The GO content was determined using HPLC (Agilent Technology 1,200 series, Germany) with an Eclipse Zorbax XDB-C 18 (Agilent, $250 \times 4.6 \mathrm{~mm}, 5 \mu \mathrm{m}$ ) analytical column and a Zorbax XDB-C18 (Agilent, 12.5 $\times 4.6 \mathrm{~mm}, 5 \mu \mathrm{m})$ guard column. Sample was then eluted at a flow rate of $1.4 \mathrm{ml} / \mathrm{min}$ with a mobile phase composed of methanol, acetonitrile, dichloromethane, and acetic acid (55:35:9.5:0.5). The freeze-dried GOZNs were kept in an aluminum foil under $-180 \mathrm{C}$ and taken out every $1,7,14$, and 42 days to determine the GO content ( $\mathrm{mg} / \mathrm{g}$ sample) remaining in GOZNs. 


\subsection{Procurement of Sprague Dawley rat}

Healthy female Sprague Dawley rats (8 weeks of age) were obtained from the Nomura Siam International Co., Ltd., Thailand. There were five rats per cage under standard laboratory conditions, with a period of $12 \mathrm{~h}$ light $/ 12 \mathrm{~h}$ dark, at $23 \pm 1{ }^{\circ} \mathrm{C}$ under $40-60 \%$ relative humidity. The animals were provided with commercial food and had tap water ad libitum. According to the National Act on the Use of Experimental Animals (Thailand) requirement, the protocols for all animals studies were reviewed and approved by the Animal Ethics Committee of Thailand Institute of Scientific and Technological Research (No. T-62021).

\subsection{Administration and observations}

The acute oral toxicity study was conducted following OECD 420 Guideline for Testing of Chemicals, acute oral toxicity-fixed dose procedure [26]. After one week of acclimatization period, the rats were 9 weeks old at the start of the study (Day 1) and had an average body weight of $222.60 \pm 3.03 \mathrm{~g}$. The animals were fasted for 16 hours with ad libitum water prior to dosing. They were administered with a single oral gavage dose of GOZNs at $2000 \mathrm{mg} \mathrm{GO} / \mathrm{kg}$ body weight. The GO content in the GOZNs for the feeding was calculated based on the HPLC assay as described above. The GOZNs powders were dissolved in distilled water and mixed well before the oral administration. Toxicity signs were recorded systematically starting the first $30 \mathrm{~min}$ and periodically during the first 24 hours, with particular attention during the first 4 hours. The surviving rats were monitored for 14 days for any changes in the general physical conditions such as appearance, fur condition, behavior, and mortality. The body weight of the animals was measured before the dosing and then weekly after (Day 1,8 , and 15). Surviving animals were sacrificed using a carbon dioxide method of euthanasia on day 15 and undergone gross necropsy.

\subsection{Statistical analysis}

All data were expressed as mean \pm SD unless otherwise stated. Statistical significance was determined by oneway analysis of variance (ANOVA) and post hoc least significant difference (LSD) test using the SPSS program (IBM SPSS Statistics 22). Statistical significance was determined at $p$-values of less than 0.05 .

\section{Equations and mathematics}

\subsection{GOZNs and their properties}

GOZNs displayed an average diameter of $311.20 \pm 3.01$ $\mathrm{nm}$. Zein is known to self-assemble as nanospheres during anti-solvent precipitation. The low PDI value, $0.10 \pm 0.02$ (lower than 0.05), displayed a highly monodispersed distribution of GOZNs in the dispersion [28]. The mean $\zeta$-potential of GOZNs was $-54.91 \pm 0.60$
$\mathrm{mV}$. This value exhibited that the freshly prepared dispersion had good colloidal stability [29]. Moreover, at above $\mathrm{pH}$ 8.0, the carboxylic groups of glutamate in zein are deprotonated to result in numerous negative charges [30]. The properties of the GOZNs prepared for the acute toxicity test in animals were in the same range as our previous study [18].

\subsection{Morphological observation}

The SEM images of the zein, GO and GOZNs are presented in Fig. 1. Zein and GO had irregular structures, while GOZNs had a spherical shape with a smooth surface. These observations were consistent with our previous study [18]. The mean diameter of particles estimated from SEM images $(218.86 \pm 0.04 \mathrm{~nm})$ was smaller than that estimation from DSL. Similar observations were reported by Zhong and Jin [31], due to the effect of freeze drying.

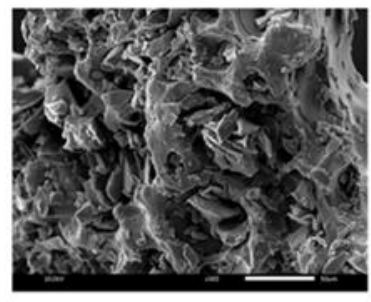

(A)

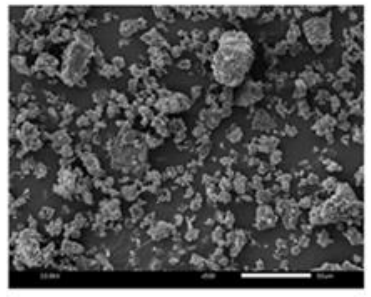

(B)

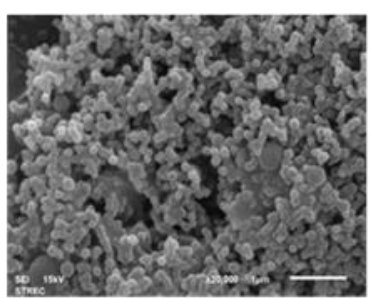

(C)

Fig. 1. Scanning electron micrographs (SEM) of zein (A), gamma oryzanol (B), and GOZNs (C). Scale bars represent 10 $\mu \mathrm{m}$ in $(\mathrm{A})$ and $(\mathrm{B})$, and $1 \mu \mathrm{m}$ in $(\mathrm{C})$.

\subsection{GO content in GOZNs}

The HPLC chromatogram used to determine GO content in the freeze-dried GOZNs powder exhibited five peaks at retention times of 15.96, 17.87, 19.20, 20.67, and $21.95 \mathrm{~min}$. GO consisted of five major compounds which were cycloartenyl ferulate $(32.65 \%), 24$-methylenecycloartanyl ferulate $(38.62 \%), \Delta 7$ campestenyl ferulate $(16.63 \%)$, campesteryl ferulate $(0.83 \%)$, and sitosteryl ferulate (9.65\%) (Fig. 2). During the storage at $-18^{\circ} \mathrm{C}$, the GO content ( $\mathrm{mg} / \mathrm{g}$ sample) in GOZNs was not significantly different on day $1,7,14$, and 42 (Fig. 3). GO content decreased from $322.69 \pm 5.67$ to $311.22 \pm 7.97$ $\mathrm{mg}-\mathrm{GO} / \mathrm{g}$-powder, which were equivalent to $\mathrm{GO}$ retention of $100 \%$ to $96.44 \%$ compared to the first day of preservation. The previously reported GO retention in GOZNs, decreasing to $90.13 \%$ when stored in a clear glass vial at $-18 \circ \mathrm{C}$ for 45 days [18]. However, free GO obtained lower GO contents (87.53\%) than both GOZNs 
samples after storage for 40 days (data do not shown). Zein could improve the stability of the encapsulated bioactive by delaying deteriorative reactions and thus enhancing chemical stability during storage [32].

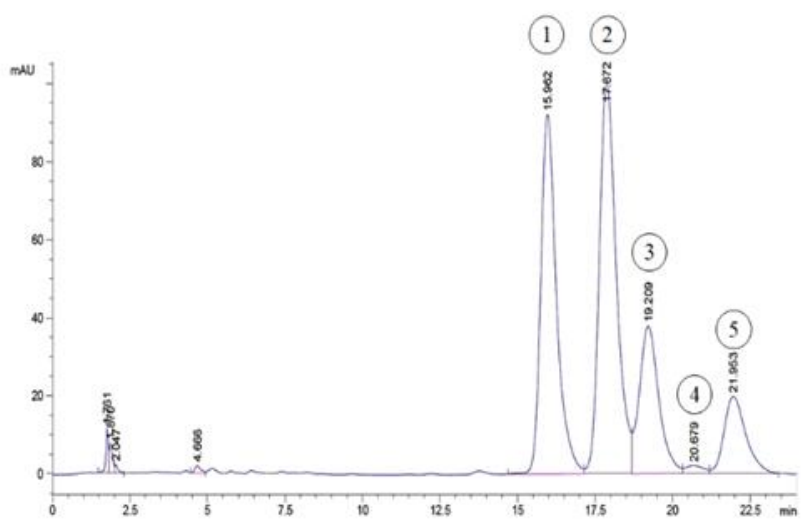

Fig. 2. HPLC chromatogram of gamma oryzanol. Peaks 1 to 5 indicate the following ferulate esters of triterpenoids: (1) cycloartenol, (2) 24-methylenecycloartanol, (3) campesterol, (4) cycloblanol, and (5) sitosteryl ferulate.

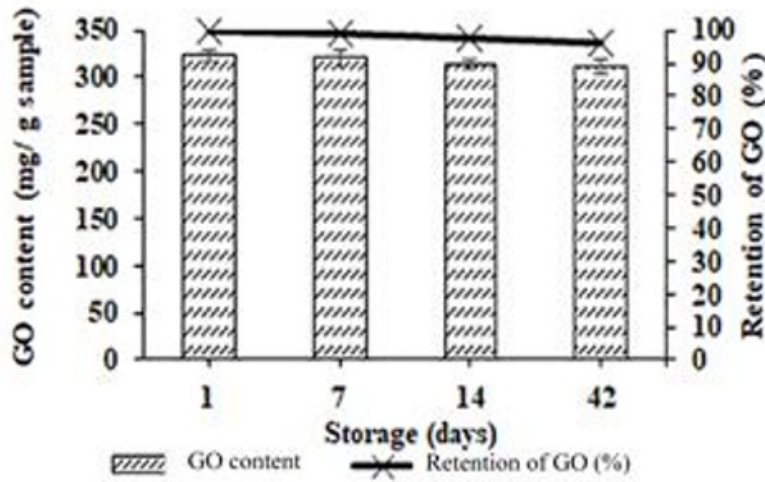

Fig. 3. Changes of GO content (mg/g sample) and \% retention of GO during storage at $-18^{\circ} \mathrm{C}$.

\section{4 Animal observation and body weight}

Behavior toxicity signs in Sprague Dawley rats were observed after a single oral dose at 2,000 $\mathrm{mg} \mathrm{GO} / \mathrm{kg}$ body weight. No changes of skin, fur, eyes, mucous membranes, respiration, circulatory system, autonomic and central nervous systems were found after the feeding. In addition, daily examination of all rats for their physical conditions showed none of the abovementioned toxicity signs, and no mortality was recorded throughout the 14-day observation period (Table 1).

Moon and co-worker [24] investigated the effects of GO in Sprague Dawley rats with the dose of 2,000 $\mathrm{mg} / \mathrm{kg}$ body weight in which no adverse effects and no histopathological changes in various organs were noticed. The same dose for GO in the GOZNs in the present study, also revealed no negative effects as acute toxicity in the after receiving GOZNs, the body weight of all tested animals increased throughout the study and demonstrated to be significantly different between day 1 and 8 , and day 8 and 15 (P < 0.05) (Fig. 4) with $15.61 \%$ and $7.42 \%$ body weight change, respectively. From the gross pathological examination, no significant abnormality of all internal organs including lungs, heart, thymus, esophagus, trachea, stomach, small intestine, large intestine, liver, spleen, pancreas, adrenals, kidneys, ovary, and uterus was observed. rats, implying that GOZNs are non-toxic.

Table 1. Toxicological effect of the acute exposure of GOZNs in Sprague Dawley rats at 2,000 mg/kg body weight.

\begin{tabular}{|c|c|}
\hline Time & Clinical signs* \\
\hline 30 min & None \\
\hline 4 hours & None \\
\hline 24 hours & None \\
\hline Up to 8 days & None \\
\hline Up to 15 days & None \\
\hline
\end{tabular}

In addition, the oral administration of GO in GOZNs at a dose limit of $2,000 \mathrm{mg} / \mathrm{kg}$ produced neither mortality nor acute toxicity signs throughout 14 days of the observation. The slight increase in body weight of the treated animals can still be accepted as normal, as it can arise due to differences in food consumption. However, the study on long-term consumption, which might increase the cytotoxic risk related to subchronic toxicity of the GOZNs in animals, would be beneficial for food applications in the future.

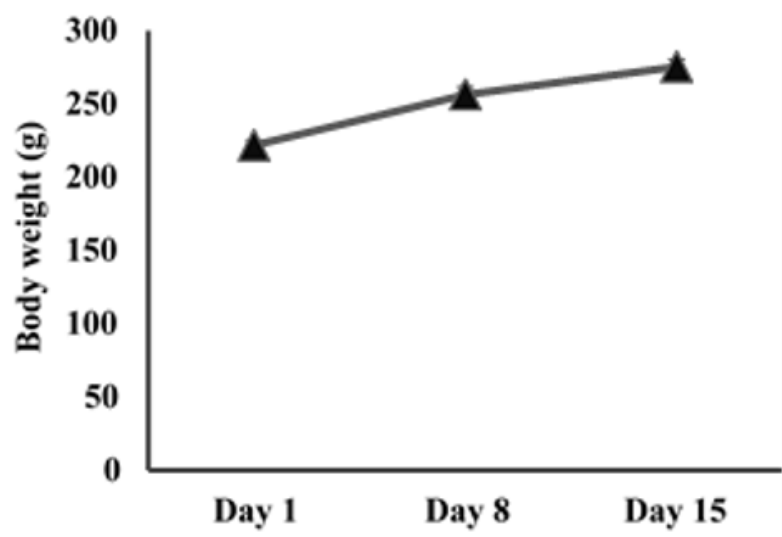

Fig. 4. Body weight (g) of female rats (age $=9$ weeks, $\mathrm{n}=5$ ) after receiving a single dose of GOZNs in an acute toxicity (at day 1).

Note: * Significantly different from the Day 1 group $(\mathrm{P}<0.05)$, ** Significantly different from the Day 8 group $(\mathrm{P}<0.05)$

\section{Conclusion}

The GOZNs prepared in this study exhibited a nanoscale diameter, low polydispersity index, and highly negative zeta-potential, as well as the good retention of $\mathrm{GO}$ during 42 -day storage at $-18^{\circ} \mathrm{C}$. The acute toxicity test of GOZNs in Sprague Dawley rats, using OECD 420 via single oral administration at $2,000 \mathrm{mg} / \mathrm{kg}$, showed no mortality and toxicity signs during the 14-day experiments, and the average body weight of rats increased during observations. The results provide 
preliminary safety information for GOZNs, which should be investigated for subchronic toxicity in the future. The present work is significant to the development of GOZNs as value-adding products using zein (corn) and GO (rice bran) from agricultural raw materials for use in nutraceuticals or food and beverage industries to promote human health.

\section{Acknowledgement}

The authors would like to thank the Thailand Institute of Scientific and Technological Research (grant number 886109) for their facilities and Thailand Research Fund, under the Research and Researchers for Industries program (grant number PHD59I0053) for their financial support.

\section{References}

1. H. Demaree-Saddler https://www.worldgrain.com/articles/14832-thailand-sees-uptick-inrice-production (2021)

2. A. Moongngarm, J. Amornpan, A. Homduang, C. Haoteng, A. Jelekovac, Rice Starch as Coating Materials for Microencapsulation of Gamma Oryzanol to Improve its Stability, American Journal of Applied Sciences, 13, 8 (2016): 900-906

3. K. A. Szcześniak, P. Ostaszewski, A. Ciecierska, T Sadkowski, Investigation of Nutriactie Phytochemical Gamma Oryzanol in Experimental Animal Models, Journal of Animal Physiology and Animal Nutrition, 100, 4 (2016): 601-617

4. A. Sukhanova, S. Bozrova, P. Sokolov, M. Berestovoy, A. Karaulov, I. Nabiev, Dependence of Nanoparticle Toxicity on their Physical and Chemical Properties, Nanoscale Research Letters, 13, 1 (2018): 1-21

5. J.S. Lee, J.S. Kim, H.G. Lee, Dependence of Nanoparticle Toxicity on their Physical and Chemical Propertiees, Colloids and Surfaces B: Biointerfaces, 70, 2 (2009): 213-217

6. M.R. Kasaai, Trends in Food Science and Technology, 79 (2018): 184-197

7. G. Spigno, F Donsì, D. Amendola, M. Sessa, G. Ferrari, D. M. De Faveri, Nanoencpsulattion Systems to Improve Solobility and Antioxidant Efficiency of a Grape Marc ExtracT into Hazelnut Paste, Journal of Food Engineering, 114, 2 (2013): 207-214

8. A. Jain, G. Sharma, V. Kushwah, G. Ghoshal, A. Jain, B. Singh, U.S. Shivhare, S. Jain, O.P. Katare, Beta Carotene-Loaded Zein Nanoparticles to Improve the Biopharmaceutical Attributes and to Abolish the Toxicity of Methotrexate: a Preclinical Study for Breast Cancer, Artificial Cells, Nanomedicine, and Biotechnology, 46, sup 1 (2018): 402-412
9. A.O. Elzoghby, M.M. Elgohary, N.M. Kamel, Implications of Protein and Peptide based Nanoparticles as Potential Vehicles for Anticancer Drug, In Advances in Protein Chemistry and Structural Biology, (2015):169-221

10. J. He, L. Wang, Z. Wei, Y. Yang, C. Wang, X. Han, Z. Nie, Vesicular Self-Assembly of Colloidal Amphiphiles in Microfluidics, ACS Applied Materials and Interfaces, 5, 19 (2013): 9746-9751

11. L.Y. Luo, B. Zhang, M. Whent, L.L. Yu, Q. Wang, Preparation and Characterization of Zein/Chitosan Complex for Encapsulation of Alpha-Tocopherol, and Its in Vitri Controlled Release Study, Colloids and Surfaces B: Biointerfaces, 85, 2 (2011): 145152

12. X.Y., Luo, M.H. Yang, F.X. Wu, L.J. Wu, L. Chen, Z. Tang, N.T. Liu, X.F. Zeng, J.L. Guan, G.H. Yuan, Vitamin D Receptor gene Bsml Polymorphism B allele, but Not BB genotype, is associated with Systemic Impus Erythematosus in a Han Chinese Population, Lupus, 21, 1 (2012): 5359

13. S. Quispe-Condori, M.D.A. Saldaña, F. Temelli, Microencapsulation of Flax Oil with zein Using Spray and Freeze Drying, LWT - Food Science and Technology, 44, 9 (2011): 1880-1887

14. N. Parris, P.H. Cooke, K.B. Hicks, Encapsulation of Essential Oils in Zein Nanospherical Particles, Journal of Agricultural and Food Chemistry, 53, 12 (2005): 4788-4792

15. V. Rungsardthong, U. Pithanthanakul, C. Puttanlek, D. Uttapap, K. Boonpisuttinant, Preparation of Puerarin Loaded Zein Nanoparticles: Characterization and Stability Study, Journal of Current Science and Technology, 11, 1 (2021): 60-70

16. S. Suwannasang, B. Thumthanaruk, Q. Zhong, D. Uttapap, C. Puttanlek, S. Vatanyoopaisarn, V. Rungsardthong, Food and Bioprocess Technology, (2021): under review

17. U. Pithanthanakul, S. Vatanyoopaisarn, B. Thumthanaruk, C. Puttanlek, D Uttapap, B. Kietthanakorn, V. Rungsardthong, Encapsulation of Fragranced in Zein Nanoparticles and Use as Fabric Softener Textile Application, Flavour and Fragrance Journal, 36, 3 (2021): 365-373

18. U. Rodsuwan, U. Pithanthanakul, K. Thisayakorn, D. Uttapap, K. Boonpisuttinant, S. Vatanyoopaisarn, B. Thumthanaruk, V. Rungsardthong, Preparation and Charecterization of Gamma Oryzanol Loaded Zein Nannoparticles and Its Improved Stability, Food Science and Nutrition, 9, 2 (2021): 616-624

19. S.S.P. Singh, M.F. Rahman, U.S.N, Murty, M. Mahboob, P. Grover, Comparative Study of Genotoxicity and Tissue Distribution of Nano and Miron Size Iron Oxide in Rats After Acute Oral Treatmente, Toxicology and Applied Pharmacology, 266, 1 (2013): 56-6 
20. B. Mangalampalli, N. Dumala, P. Grover, Genotoxicity, Biochemical, and Biodistribution Studies of Magnesium Oxide Nano and Microparticles in Albino Wistar Rats After 29 Day Repeated Oral Exposure , Toxicology and Pharmacology, 90 (2017): 170-184

21. K. Pan, Q. Zhong, Annual Review of Food Science and Technology, 7 (2016): 245-266

22. C.D. Walkey, J.B. Olsen, H. Guo, A. Emili, W.C. Chan, Nanoparticle Size and Surface Chemistry Determine Serum Protein Adsorption and Macrophage Uptake, Journal of the American Chemical Society, 134, 4 (2012): 2139-2147

23. H.K. Biesalski, L.O Dragsted, I. Elmadfa, R. Grossklaus, M. Müller, D. Schrenk, P. Walter, P. Weber, Bioactive Compounds: Definition and Assessment of Activity, Nutrition, 25, 11-12 (2009): 1202-1205

24. S.H. Moon, D. Kim, N. Shimizu, T. Okada, S. Hitoe, H. Shimoda, Ninety-Day Oral Toxicity Study of Rice Derived Gamma Oryzanok in Sprague Dawley Rats, Toxicology Reports, 4 (2017): 9-18

25. F. Andersen, International Journal of Toxicology, 25, Sup 2 (2006): 91-120

26. OECD Guideline for Testing of Chemicals, Section 4, Health Effect. Test No 420; Acute Oral
Toxicity-Fixed Dose Procedure, Paris: Organization for Economic Corporation and Development (2001).

27. R.L. Lipnick, J.A. Cotruvo, R.N. Hill, R.D. Bruce, K.A. Stitzel, A.P. Walker, I. Chu, M. Goddard, L. Segal, J.A. Springer, R.C. Myers, Food and Chemical Toxicology. 33, 3 (1995): 223-23

28. M. Danaei, M. Dehghankhold, S. Ataei, F. Hasanzadeh Davarani, R. Javanmard, A. Dokhani, S. Khorasani, M.R. Mozafari, Impact of Particle Size and Polydispersity Index on the Clinical Application of Lipidic Nanocarrier Systems, Pharmaceutics, 10, 2 (2018): 57

29. E. Joseph, G. Singhvi, Nanomaterials for Drug Delivery and Therapy, (2019): 91-116

30. B. Zhang, Y. Luo, Q. Wang, Effect of Acid and Base Treatments on Structural, Rheological, and Antioxidant Properties of Alpha-Zein, Food Chemistry, 124, 1 (2011): 210-220

31. Q. Zhong, M. Jin, Zein nanoparticles Produced by Liquid-Liquid Dispersion, Food Hydrocolloids, 23, 8 (2009): 2380-2387

32. S. Torres-Giner, A. Martinez-Abad, M.J. Ocio, J.M. Lagaron, Stabilization of a Nutraceutical Omega-3 Fatty Acid by Encapsulation in Electrosprayed Zein Prolamine, Journal of Food Science, 75, 6 (2010): N69-N79 